\title{
Validation of the Beliefs about Medicines Questionnaire and Self-Efficacy for Appropriate Medication Use Scale to measure pharmacological treatment adherence in patients with inflammatory bowel disease
}

\author{
Katya Estefanía Bozada-Gutiérrez, ${ }^{1}$ Ana Fresán-Orellana² and Jesús K. Yamamoto-Furusho ${ }^{1 *}$ \\ 'Secretaría de Salud, Instituto Nacional de Ciencias Médicas y Nutrición "Salvador Zubirán", Inflammatory Bowel Disease Clinic; ${ }^{2}$ Secretaría de \\ Salud, Instituto Nacional de Psiquiatría "Ramón de la Fuente Muñiz", Clinical Research Sub-directorate. Ciudad de México, Mexico
}

\begin{abstract}
Introduction: Treatment adherence is crucial in inflammatory bowel disease (IBD) to prevent relapses and complications. In Mexico, there is not a validated tool to assess adherence in patients with IBD. Objective: To translate the beliefs about medicines questionnaire (BMQ) and self-efficacy for appropriate medication use scale (SEAMS) instruments, as well as to determine their validity, reliability and sensitivity in IBD-diagnosed Mexican patients. Method: After informed consent was obtained, 149 IBD-diagnosed patients were included. The instruments were translated into Spanish and were subsequently applied during medical consultation. For SEAMS, exploratory factorial analysis and ROC curve analysis were carried out and Cronbach's alpha was determined; for the BMQ, Cohen's kappa coefficient and its predictive capacity were employed. Results: Seventy-five women (50.3\%) were included, with an average age of 44 years. The SEAMS scale showed a single factor that was highly reliable (Cronbach's alpha $=0.92)$ and a cutoff point of 33 to identify adherent patients. The "adherence" and "recall barrier" dimensions of the BMQ were adequate adherence predictors. Conclusions: The SEAMS and BMQ Spanish versions are valid for measuring self-efficacy and barriers to pharmacological treatment adherence in Mexican patients with IBD.
\end{abstract}

KEY WORDS: Adherence. Self-efficacy. Barriers. Pharmacological treatment. Inflammatory bowel disease.

\section{Introduction}

Inflammatory bowel disease (IBD) includes chronic idiopathic ulcerative colitis (CIUC) and Crohn's disease, ${ }^{1}$ which are chronic inflammatory diseases where life-long medical treatment is essential, and pharmacological treatment adherence (PTA) is therefore of utmost importance. The World Health Organization ${ }^{2}$ defines adherence as: "the extent to which a person's behavior - taking medication, following a diet and executing lifestyle changes corresponds, with agreed recommendations from a health care provider".

Despite the availability of numerous effective pharmacological treatments for the management of IBD, patients' lack of adherence to prescribed medications represents the greatest hindrance to therapeutic effectiveness and causes negative outcomes in patients, which result in increased disease activity or decreased treatment effectiveness, ${ }^{3}$ increased risk of relapse, ${ }^{4}$ higher risk of hospitalization and hospital stay prolongation, ${ }^{5}$ which in turn cause greater use of health resources and poorer quality of life. ${ }^{6}$

Several investigations have assessed the role of PTA in patients with IBD. A recent systematic review concluded that the lack of adherence to 5-aminosalicylate drugs increases the risk of relapse in patients with CIUC. ${ }^{7}$ In addition, 5-aminosalicylates continued use of may reduce the risk for developing colorectal cancer in this group of patients. ${ }^{8}$ In a 10-year retrospective study in the United States that included
Correspondence:

*Jesús K. Yamamoto-Furusho

E-mail: kazuofurusho@hotmail.com
Date of reception: 23-05-2018

Date of acceptance: 16-11-2018

DOI: 10.24875/GMM.M19000243
Gac Med Mex. 2019;155:112-117

Contents available at PubMed www.gacetamedicademexico.com 
13,062 patients with CIUC, with a median follow-up of 6.1 years, patients with low adherence to oral mesalazine (5-aminosalicylic acid) were observed to experience a significant increase in the risk for CIUC outbreaks in comparison with highly adherent subjects $(\mathrm{RR}=2.8, \mathrm{p}<0.001) .{ }^{9}$

There are two types of methods to measure treatment adherence: directly and indirectly; ${ }^{10}$ however, none has been considered to be a gold standard per se. Indirect methods, mainly self-administered questionnaires, have the advantage of being simple, practical and reproducible in medical practice; they are the best way to approach patient PTA by the doctor. In a systematic review, Nguyen et al. ${ }^{11}$ identified 43 validated scales to measure PTA, out of which the most widely used are the Morisky-Green scale, ${ }^{12}$ MARS, ${ }^{13}$ the Beliefs about Medicines Questionnaire $(B M Q)^{14}$ and the Self-Efficacy for Appropriate Medication Use Scale (SEAMS). ${ }^{15}$

The SEAMS scale,$^{15}$ designed by Bandura et al., ${ }^{16}$ measures self-efficacy, the basis of which is patient conviction, certainty or confidence on being able to successfully implement a specific behavior to achieve a particular outcome: ${ }^{17}$ PTA. On the other hand, BMQ ${ }^{14}$ focuses on identifying concerns, beliefs and negative barriers about treatment that influence on PTA, which has been validated in different pathologies: asthma, ${ }^{18}$ human immunodeficiency virus infection, ${ }^{19}$ psychiatric disorders, ${ }^{20}$ rheumatoid arthritis ${ }^{21}$ and IBD. ${ }^{22}$

Recognizing the negative impact of the lack of PTA on IBD clinical course, the need arose for having validated instruments that help gastroenterologists to evaluate it.

The purpose of the present study is to translate and validate the SEAMS and BMQ scales to measure adherence, self-efficacy and barriers to pharmacological treatment in Mexican patients with IBD.

\section{Method}

The present study was approved by the research and ethics committees of Instituto Nacional de Ciencias Médicas y Nutrición "Salvador Zubirán", where it was carried out, in accordance with the standards of good clinical practice.

From August 1, 2017 to January 31, 2018, patients of between 18 and 70 years of age of the IBD Clinic of the Institute, who had a histopathology-confirmed IBD diagnosis and who had been on follow-up for more than six months, under pharmacological treatment at the time of the study, who knew how to read and write, were included. Those who were not under pharmacological treatment or who delivered the instruments incompletely were excluded.

Once permission from SEAMS and BMQ original authors was obtained, the instruments were translated and adapted into Spanish according to the recommended direct and inverse translation methodology. ${ }^{23}$ The recruitment of patients began after the translation process was completed.

\section{Evaluation instruments and procedure}

- SEAMS: ${ }^{15}$ 13-item, self-administered instrument that measures patients' self-efficacy with regard to taking their medications. Patients indicate, according to 13 situations asked by the items, the level of confidence on correctly taking their medications: 1 , not at all confident; 2 , somewhat confident; 3 , very confident. The sum of results ranges from 13 to 39 points, where higher scores indicate optimal self-efficacy for taking the pharmacological treatment.

- BMQ:14 17-item, standardized scale that assesses specific concerns about the medication the person is taking and the beliefs about its importance. The original version is composed of two sections: BMQ-general (seven items) and BMQ-specific (10 items). The items are assessed using a five-point scale that ranges from 1, strongly disagree, to 5 , strongly agree. BMQ-general includes the overuse and harm subscales and, BMQ-specific, the belief in the necessity of the medication and concern about the consequences of the medication regarding IBD pharmacological treatment subscales. At the end, the sum of all four dimensions generates a score between 0 to 4 points, where 0 is treatment adherence and no barriers to adherence, and 4, non-adherence and presence of any of the three barriers to adherence (beliefs, forgetfulness and access to medication).

- Visual Analogue Scale (VAS): the results of the SEAMS and BMQ scales were compared with the treating physician assessment during the patient visit, who was asked direct questions about taking the drugs and PTA, together with an assessment of the disease global activity level (with clinical and biochemical parameters), where lack of adherence is considered to predispose to higher IBD activity and relapses, ${ }^{24}$ with adherence being measured with the VAS, which was regarded as the gold standard. The VAS goes from 0 
to 10 , where the patients were classified as adherent when the treating physician rated with a score of 9 or 10 and as non-adherent when the scores were $\leq 8 .^{25}$

\section{Statistical analysis}

Demographic and clinical characteristics were described with frequencies and percentages for categorical variables and means and standard deviations (SD) were used for continuous variables.

To test SEAMS clinimetric properties, the following procedures were followed:

- A correlation matrix was generated for the items; those with a coefficient lower than 0.20 would be eliminated from further analysis.

- An exploratory factor analysis with varimax rotation was carried out to prove the construct validity; items with communalities lower than 0.40 were excluded.

- Internal consistency was determined using Cronbach's alpha coefficient.

- A receiver operating characteristic (ROC) curve was plotted to determine the most appropriate SEAMS score in terms of sensitivity and specificity.

For $\mathrm{BMQ}$ :

- Cohen's kappa coefficient was employed to determine the concordance between the adherence report of the instrument and the classification made by treating physicians.

- The predictive capacity of BMQ's four dimensions was defined, with different levels of adherence according to clinical assessment.

\section{Results}

One-hundred and forty-nine patients were included, out of whom $50.3 \%(n=75)$ were females. Patients' average age was 44.2 years $(S D=13.6$, range $=20$ 78). Most included patients had a CIUC diagnosis $(81.2 \%, n=121)$, whereas $18.8 \%(n=28)$ had been diagnosed with Crohn's disease. All patients were under pharmacological treatment at the time of their inclusion in the study (mesalazine, $77.9 \%, n=116$, azathioprine, $36.9 \%, \mathrm{n}=55$; steroids, $33.6 \%$; and sulfasalazine $8.7 \%, n=13$, mainly). According to the VAS, average adherence score assigned by the treating physicians was $7.8(\mathrm{SD}=1.4,4-10) ; 45.6 \%(\mathrm{n}=68)$ of patients were classified as adherent and $54.4 \%$ $(\mathrm{n}=81)$ as non-adherent.
Table 1. Factor analysis of the Self-Efficacy for Appropriate Medication Use Scale (SEAMS) in Mexican patients with inflammatory bowel disease

\begin{tabular}{|c|c|}
\hline Item & Factor 1 \\
\hline 1. If you take several different medicines each day. & 0.669 \\
\hline 2. If you take medicines more than once a day. & 0.774 \\
\hline 3. If you are away from home. & 0.819 \\
\hline 4. If you have a busy day planned. & 0.846 \\
\hline 5. If they cause some side effects. & 0.784 \\
\hline 6. If no one reminds you to take the medicine. & 0.761 \\
\hline 7. If the schedule to take the medicine is not convenient. & 0.690 \\
\hline 8. If your normal routine gets messed up. & 0.661 \\
\hline 9. If you are not sure how to take the medicine. & 0.730 \\
\hline $\begin{array}{l}\text { 10. If you are not sure what time of the day to take your } \\
\text { medicine. }\end{array}$ & 0.732 \\
\hline 11. If you are feeling sick (like having a cold or the flu). & 0.720 \\
\hline $\begin{array}{l}\text { 12. If you get a refill of your old medicines and some of } \\
\text { the pills look different than usual. }\end{array}$ & 0.637 \\
\hline 13. If your doctor changes your medicines. & 0.712 \\
\hline Eigenvalue & 7.03 \\
\hline Variance & 54.14 \\
\hline
\end{tabular}

\section{SEAMS, clinimetric properties}

The correlation ranges of all 13 SEAMS items were between 0.58 and 0.80 and, therefore, no item was eliminated and all were included in the analysis. The results showed that one single factor (Table 1) explained $54.1 \%$ of the variance. The model was regarded as adequate, according to a Kaiser-Meyer-Olkin value of 0.91 and a highly significant result on Bartlett's test of sphericity $(p<0.001)$. This factor is highly reliable (Cronbach's alpha $=0.92$ ).

When evaluating SEAMS best cut-off point to identify adherent patients, a score of 33 was found to show more stable sensitivity and specificity values, with an area under the curve value of 0.92 (Figure 1), which is indicative of SEAMS adequate discriminating capacity.

\section{$B M Q$, clinimetric properties}

In BMQ first assessment dimension, adherence, $45.6 \%(n=68)$ of patients were classified as adherent. In the following dimensions, focused on the assessment of barriers to adherence, $28.9 \%(n=43)$ were observed to report negative beliefs about medications, 


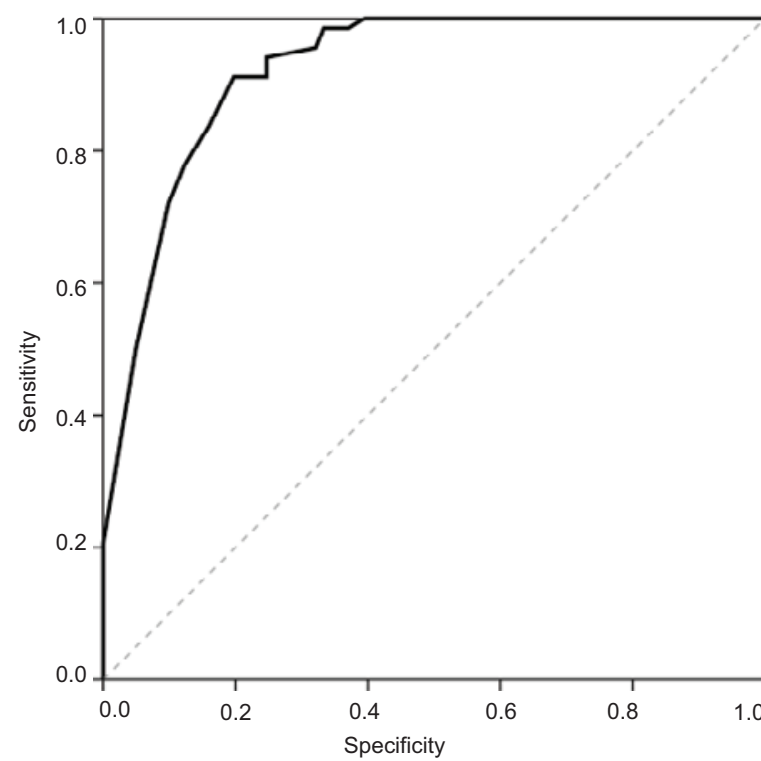

\begin{tabular}{|l|c|c|c|c|}
\hline Cutoff point & Sensitivity & Specificity & $\begin{array}{c}\text { Positive } \\
\text { predictive } \\
\text { value }\end{array}$ & $\begin{array}{c}\text { Negative } \\
\text { predictive } \\
\text { value }\end{array}$ \\
\hline 30 points & 0.96 & 0.68 & 0.71 & 0.95 \\
\hline 31 points & 0.94 & 0.75 & 0.76 & 0.94 \\
\hline 32 points & 0.91 & 0.75 & 0.76 & 0.91 \\
\hline 33 points & 0.91 & 0.80 & 0.79 & 0.92 \\
\hline
\end{tabular}

Figure 1. SEAMS scale cutoff points. Sensitivity, specificity and predictive values.

Table 2. Belief Medicines Questionnaire (BMQ) dimensions predictive capacity

\begin{tabular}{|c|c|c|c|c|}
\hline VAS & Sensitivity & Specificity & Positive predictive value & Negative predictive value \\
\hline \multicolumn{5}{|c|}{ BMQ-Adherence } \\
\hline Adherence 7 & 1.00 & 0.36 & 0.57 & 1.00 \\
\hline Adherence 8 & 1.00 & 0.60 & 0.68 & 1.00 \\
\hline Adherence 9 & 1.00 & 1.00 & 1.00 & 1.00 \\
\hline \multicolumn{5}{|c|}{ BMQ-Negative beliefs about medicines } \\
\hline Adherence 7 & 0.90 & 0.42 & 0.79 & 0.62 \\
\hline Adherence 8 & 0.82 & 0.70 & 0.87 & 0.61 \\
\hline Adherence 9 & 0.58 & 0.84 & 0.90 & 0.44 \\
\hline \multicolumn{5}{|c|}{ BMQ-Recall barrier } \\
\hline Adherence 7 & 0.97 & 0.35 & 0.58 & 0.93 \\
\hline Adherence 8 & 0.90 & 0.54 & 0.64 & 0.86 \\
\hline Adherence 9 & 0.86 & 0.91 & 0.90 & 0.88 \\
\hline \multicolumn{5}{|c|}{ BMQ-Access to medication barrier } \\
\hline Adherence 7 & 0.75 & 0.14 & 0.48 & 0.34 \\
\hline Adherence 8 & 0.64 & 0.30 & 0.49 & 0.45 \\
\hline Adherence 9 & 0.47 & 0.56 & 0.53 & 0.51 \\
\hline
\end{tabular}

VAS $=$ visual analogue scale, VAS $9=$ adequate adherence, VAS 7-8 $=$ moderate adherence.

$47.7 \%(n=71)$ barriers to remembering taking the medications and $51 \%(n=76)$, barrier of access to medications. BMQ Cohen's kappa coefficient in the assessment of adherence, in contrast with the treating physicians' report was $1.0(p<0.001)$, indicating ideal concordance.
Table 2 describes the sensitivity, specificity and predictive values of the BMQ dimensions for identifying patients with adequate adherence according to the treating physicians' report (VAS $\geq 9$ ) and those with moderate adherence (VAS = 7- 8). The BMQ adherence dimension had ideal values for identification of 
adequate adherence, although its specificity and positive predictive value for identifying patients with moderate adherence were decreased. On the other hand, the negative beliefs about medications and access to medications barrier dimensions indicated inadequate values for the prediction of adequate and moderate adherence. The recall barrier dimension had adequate values to identify patients with adequate adherence, unlike those with moderate adherence, whose specificity and positive predictive values were low.

\section{Discussion}

Inadequate PTA negatively impacts on several chronic diseases' clinical evolution, especially in IBD, where taking medications for life is essential to maintain remission. Lack of PTA has been described to lead to fatal clinical outcomes, as well as increased hospital admissions, which negatively impact on patient quality of life. ${ }^{4}$

The presented study is the first one in Mexico on the validation and translation of two useful instruments to monitor PTA in patients with IBD. According to our results, the translated SEAMS scale factor analysis indicated that the entire instrument pointed at the self-efficacy dimension, unlike the original version, ${ }^{15}$ where the factor analysis revealed two medication-related self-efficacy dimensions: self-efficacy to take medications in difficult circumstances, such as when patients are busy, away from home or have to take multiple medications, and self-efficacy to take medications in uncertain or changing circumstances, such as when the patient is not sure about how to take them. Similar results were obtained by other groups that translated and validated the instruments. On the other hand, Pedrosa et al. $^{26}$ validated SEAMS in a Brazilian population, where the analysis discriminated for two factors as in the original version.

The best cut-off point to discriminate adherent patients on the SEAMS scale was 33 points, based on which the area under the curve was 0.92 (sensitivity of $91 \%$ and specificity of $80 \%$ ), which indicated adequate discriminant capacity of the instrument. In China, Dong et al. ${ }^{27}$ validated and translated the SEAMS scale into the Chinese language in patients with cerebral infarction; the best cutoff point was 32 points, similar to ours.

On the other hand, according to our BMQ scale factor analysis, only two of the four dimensions of the instrument were predictors of adherence in patients with IBD: adherence in general and recall barriers ( $86 \%$ sensitivity and $91 \%$ specificity); the negative beliefs about medication and access to medications barriers dimensions showed inadequate values for the prediction of adequate adherence.

In the ALIGN study, ${ }^{28}$ PTA was measured using the BMQ scale, whereby a significant proportion of patients $(52.4 \mathrm{~m} \%)$ was described to have negative beliefs about medication and, nevertheless, showed treatment adherence despite this barrier, which are similar findings to ours. With the BMQ questionnaire for measuring PTA, Castro et al. ${ }^{29}$ found that the low-adherence dimension was associated with high levels of concern about the medication used to control IBD, while high scores regarding potential harm of medications in general were significantly associated with lack of adherence.

Among the limitations of this study, the instruments' validation is identified to have been carried out in a single national reference center, in a tertiary-care hospital, which hinders generalization of the results to the entire Mexican IBD-diagnosed population. Notwithstanding, the BMQ and SEAMS instruments validated in the Mexican population with IBD provide key information on PTA that will allow the creation of strategies to optimize patient treatment adherence, with a consequent improvement of clinical outcomes.

\section{Conclusions}

The Spanish translation and validation of the SEAMS and BMQ scales showed adequate clinimetric properties, which is indicative of reliability to measure PTA in Mexican patients with IBD.

\section{References}

1. Abraham C, Cho JH. Inflammatory bowel disease. N Engl J Med. 2009; 361:2066-2078.

2. Sabaté E. Adherence to long-term therapies: evidence for action. Suiza: World Health Organization; 2003.

3. Mårdby AC, Åkerlind I, Jörgensen T. Beliefs about medicines and self-reported adherence among pharmacy clients. Patient Educ Couns. 2007; 69:158-164

4. Kane S V, Robinson A. Understanding adherence to medication in ulcerative colitis-innovative thinking and evolving concepts. Aliment Pharmacol Ther. 2010:32:1051-1058

5. Mitra D, Hodgkins P, Yen L, Davis KL, Cohen RD. Association between oral 5-ASA adherence and health care utilization and costs among patients with active ulcerative colitis. BMC Gastroenterol. 2012;12:132.

6. Kane SV, Chao J, Mulani PM. Adherence to infliximab maintenance therapy and health care utilization and costs by Crohn's disease patients. Adv Ther. 2009;26:936-946.

7. Higgins PD, Rubin DT, Kaulback K, Schoenfield PS, Kane SV. Systematic review: impact of non-adherence to 5-aminosalicylic acid products on the frequency and cost of ulcerative colitis flares. Aliment Pharmacol Ther. 2009:29:247-257.

8. Van Staa TP, Card T, Logan RF, Leufkens HG. 5-aminosalicylate use and colorectal cancer risk in inflammatory bowel disease: a large epidemiological study. Gut. 2005:54:1573-1578.

9. Khan N, Abbas AM, Bazzano LA, Koleva YN, KrouseldWood M. Longterm oral mesalazine adherence and the risk of disease flare in ulcerative colitis: nationwide 10 -year retrospective cohort from the 
veterans affairs healthcare system. Aliment Pharmacol Ther. 2012;36: 755-764.

10. Osterberg L, Blaschke T. Adherence to medication. N Engl J Med. 2005; 353:487-497.

11. Nguyen TMU, La-Caze A, Cottrell N. What are validated self-report adherence scales really measuring? A systematic review. Br J Clin Pharmacol. 2014;77:427-445.

12. Morisky DE, Green LW, Levine DM. Concurrent and predictive validity of a self-reported measure of medication adherence. Med Care. 1986:24:67-74.

13. Horne R, Weinman J. Self-regulation and self-management in asthma: exploring the role of illness perceptions and treatment beliefs in explaining non-adherence to preventer medication. Psychol Heal. 2002;17:17-32.

14. Horne R, Weinman J, Hankins M. The beliefs about medicines questionnaire: the development and evaluation of a new method for assessing the cognitive representation of medication. Psychol Heal. 1999;14:1-24.

15. Risser J, Jacobson TA, Kripalani S. Development and psychometric evaluation of the self-efficacy for Appropriate Medication Use Scale (SEAMS) in low-literacy patients with chronic disease. 2007;15:203-219.

16. Bandura A. Self-efficacy mechanism in human agency. Am Psychol. 1982;37:122.

17. Burke LE, Dunbar-Jacob JM, Hill MN. Compliance with cardiovascular disease prevention strategies: a review of the research. Ann Behav Med. 1997;19:239-263.

18. Perpiñá-Tordera M, Martínez-Moragón EM, Belloch-Fuster A, LlorisBayo A, Pellicer-Císcar C. Spanish asthma patients' beliefs about health and medicines: validation of 2 questionnaires. Arch Bronconeumol. 2009;45:218-223.

19. Fall E, Gauchet A, Izaute M, Horne R, Chakroun N. Validation of the French version of the Beliefs about Medicines Questionnaire (BMQ) among diabetes and HIV patients. Rev Eur Psychol Appliquée/European Rev Appl Psychol. 2014;64:335-343

20. Jónsdóttir H, Friis S, Horne R, Pettersen KI, Reikvam Å, Andreassen OA Beliefs about medications: measurement and relationship to adherence in patients with severe mental disorders. Acta Psychiatr Scand. 2009; 119:78-84.

21. Neame R, Hammond A. Beliefs about medications: a questionnaire survey of people with rheumatoid arthritis. Rheumatology (Oxford). 2005; 44:762-767.

22. Horne R, Parham R, Driscoll R, Robinson A. Patient's attitudes to medicines and adherence to maintenance treatment in inflammatory bowel disease. Inflamm Bowel Dis. 2009:15:837-844.

23. Pan $Y$, De la Puente $M$. Census bureau guideline for the translation of data collection instruments and supporting materials: documentation on how the guideline was developed. Surv Methodol. 2005;6.

24. Bluett J, Morgan C, Thurston L, Plant D, Hyrich KL, Morgan AW, et al. Impact of inadequate adherence on response to subcutaneously administered anti-tumour necrosis factor drugs: results from the Biologics in Rheumatoid Arthritis Genetics and Genomics Study Syndicate cohort. Rheumatology (Oxford). 2014;54:494-499.

25. Giordano TP, Guzmán D, Clark R, Charlebois ED, Bangsberg DR. Measuring adherence to antiretroviral therapy in a diverse population using a visual analogue scale. HIV Clin Trials. 2004:5:74-79.

26. Pedrosa RB, Rodrigues RC, Oliveira HC, Alexandre NM. Construct validity of the Brazilian version of the Self-Efficacy for Appropriate Medication Adherence Scale. J Nurs Meas. 2016;24:18-31.

27. Dong XF, Liu YJ, Wang AX, Lv PH. Psychometric properties of the Chinese version of the Self-Efficacy for Appropriate Medication Use Scale in patients with stroke. Patient Prefer Adherence. 2016;10:321-327.

28. Michetti P, Weinman J, Mrowietz U, Smolen J, Louis E, Peyrin-Biroulet L, et al. Impact of treatment-related beliefs on medication adherence in immune-mediated inflammatory diseases: results of the global ALIGN study. Adv Ther. 2017:34:91-108.

29. De Castro ML, Sanromán L, Martín A, Martínez N, Hernández V, Del Campo V, et al. Valoración de la adhesión terapéutica en la enfermedad inflamatoria intestinal. Comparación entre una escala de autoevaluación y un índice farmacéutico de dispensación de medicamentos. Rev Esp Enferm Dig. 2017;109:542-551. 